\title{
Synthesis and Characterization of APG-12
}

\author{
Jigang Wang1, Yongle Xin'1, Danting Fan², Sitong Chen ${ }^{1}$ \\ ${ }^{1}$ EOR Key Laboratory of the Ministry of Education, Northeast Petroleum University, Daqing, China \\ ${ }^{2}$ The Fourth Oil Extraction Plant of Daqing Oilfield Company Limited, Daqing, China \\ Email: wangiigang9999@163.com, 315463795@qq.com, 601345607@qq.com, 783669536@163.com
}

Received 20 October 2014; revised 22 November 2014; accepted 10 December 2014

Copyright (C) 2015 by authors and Scientifisc Research Publishing Inc.

This work is licensed under the Creative Commons Attribution International License (CC BY).

http://creativecommons.org/licenses/by/4.0/

(c) (i) Open Access

\begin{abstract}
APG-12 was synthesized from n-dodecyl alcohol and glucose in catalysis system of p-Toluenesulfonic and phosphoric acid. Using the method of direct glycosidation, this paper discusses the impact of raw material ratio, catalyst dosage and reaction temperature on the synthesis. It is concluded that the synthesis of APG-12 best reaction process condition is the molar ratio of alcohol and glucose 7:1; the reaction temperature is $115^{\circ} \mathrm{C}$ and the amount of catalyst is $1.3 \%$, controlling the reaction pressure in $3-4 \mathrm{kPa}$. The resulting products are analyzed by infrared spectroscopy.
\end{abstract}

\section{Keywords}

APG-12, Glucose, N-Dodecyl Alcohol, Infrared Spectroscopy

\section{Introduction}

In recent years, because of a lack of resources, we must search for new renewable resources. Synthesis of alkyl glycoside's raw material is a renewable resource. Its synthesis has a far-reaching significance in future society's energy development and utilization. Methods for preparing alkyl glycosides are glycosidation indirect method, direct glycosidation method, fischer method, starch degradation, mold method, tin tetrachloride catalysis method and so on. Preparation of APG-12 is currently commonly used for the direct glycosidation method. This method prepares APG-12 by glucose and long carbon chain alcohol directly. The advantage of this method is low production cost, high product purity and little by-products. Through the single factor experiment to study the effects of catalyst type and the amount of sugar alcohol ratio, reaction temperature and pressure on the experimental results, the paper ascertains the influence of various factors on the reaction.

\section{Experimental Section}

\subsection{Experimental Drugs and Laboratory Equipment}

Experimental drugs: n-dodecyl alcohol, glucose, p-Toluenesulfonic, phosphoric acid, Anhydrous copper sulfate, hydrogen peroxide, sodium hydroxide, concentrated sulfuric acid, potassium sodium tartrate. 
Laboratory equipment: Type BZY-2 automatic surface tension meter, VERTEX-55 infrared spectrometer, GJ-2-type electric mixer, NDJ-99 rotary viscometer, DK-98-1 type low temperature water bath, AE-200 electronic balance, ZK-1-type vacuum pump.

\subsection{Experimental Method}

To a volume of alcohol, glucose (slowly and portionwise) was added to the flask with a reflux condenser, a thermometer, electrical stirrer, and a vacuum system. Heating in an oil bath to a certain temperature, adding a certain amount of catalyst after stir and controlling an appropriate time and pressure within the four-neck flask until the reaction liquid was made into a white turbid supernatant liquid, then the distilled liquid was collected. Reaction after a period of time, we removed a volume of the liquid reaction mixture, neutralizing $\mathrm{pH}$ to 7 - 8 . We add Fehling reagent into the mixture, and heat it in a boiling water bath for 2 minutes. If a lot of redbrick precipitate is generated, the synthesis reaction is not completely finished. When the solution temperature fell to $60^{\circ} \mathrm{C}$, we add $5 \%$ sodium hydroxide solution under atmospheric pressure. The solution was neutralized to $\mathrm{pH}=$ 8. Oligomeric sugars were removed by filtration. Excess alcohol during the reaction was removed by vacuum distillation under lower temperature. Then, we use solvent extraction separation, getting crude product. The crude product was formulated to $50 \%$ aqueous solution of the APG-12, adding the $5 \% \mathrm{H}_{2} \mathrm{O}_{2}$ and bleaching 90 min under at $60^{\circ} \mathrm{C}$. A pale yellow viscous product was obtained [1]-[5].

\section{Results and Discussion}

\subsection{Effect of the Molar Ratio of Alcohol to Glucose}

This experiment adopts a reversible reaction. In order to make the rate of reaction fast and right, experiments generally use a high amount of alcohol solution. The higher dosage of alcohol is, the smaller polymerization degree (DP) value of synthetic products is and the better heat and mass transfer of the reaction system are. However, high dosage of alcohol will cause the subsequent refining complex of the synthetic products, and waste of resources. In our study, the molar ratio of alcohol and glucose were: 3:1, 4:1, 5:1, 6:1, 7:1, 8:1 and 9:1. The effect of the ratio of Alcohol and glucose in APG-12 synthesis is studied. We find out the best ratio of them. The results are show in Figure 1.

As can be seen from Figure 1, when the mole ratio of alcohol and glucose increases, the clarification time of reaction liquid became shorter and yield increased first and then decreased. When the ratio of alcohol and glucose was 9:1, production rate decreased to 92.4\%. The low ratio led to low yield. Because mass transfer and diathermancy became weaker as the viscosity of the reaction system became large. Glucose occurred to auto-polymerization, the high ratio of alcohol and glucose lead to high yield. Because catalyst is diluted by a large of alcohol, the catalyst concentration down is extremely low, thus reducing the reaction speed and prolonging the synthesis reaction's time. This suggests that the amount of alcohol is not the more the better. The molar ratio of

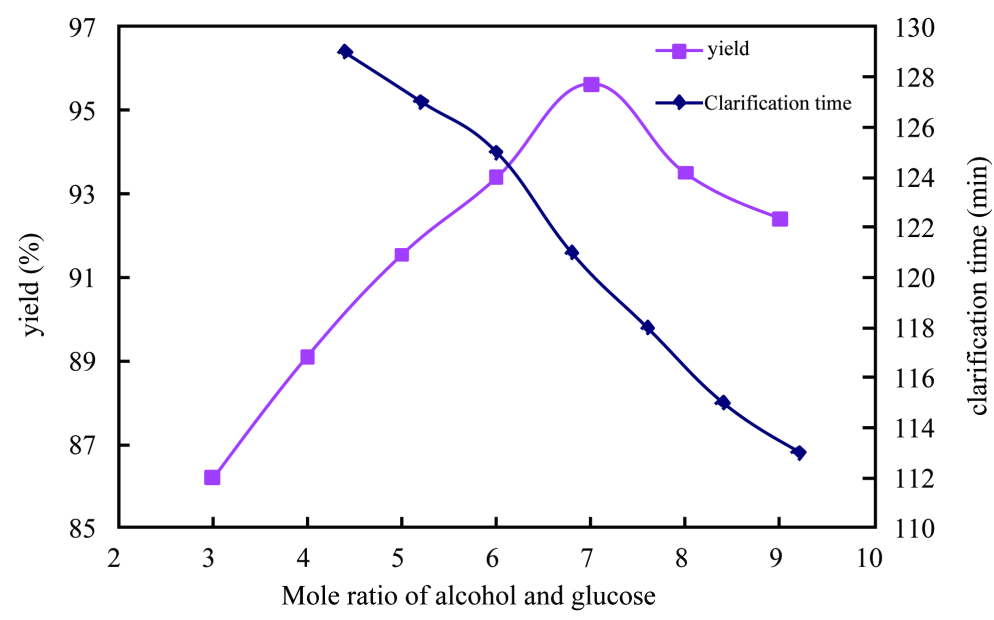

Figure 1. The effect of molar ratio of alcohol and glucose to the yield. 
alcohol and glucose 7:1 is the most appropriate.

\subsection{The Effect of Catalyst}

In order to realize the glycosidation reaction smoothly, reaction rate was accelerated and this reaction uniformly and completely reacted and didn't coke. Having high conversion rate, we need to develop a suitable binary catalyst, synthesizing APG-12 with direct glycosidation. Different types of catalysts which affect product characteristics are shown in Table 1.

Chapter 1 measured APG-12 performance under the mole ratio of alcohol and glucose which is 7:1. Reaction temperature is $115^{\circ} \mathrm{C}$. Catalyst occupies $1.3 \%$ in reaction system. The experiment data explain that acid even has different catalytic properties. Concentrated sulfuric acid had high oxidability. Excess concentrated sulfuric acid made reactant oxidized. The color of product deepened. Glucose was carbonized. Choosing property which 4-toluene sulfonic acid and phosphoric acid compounded was best because relative catalytic activity of 4-toluene sulfonic acid was better.

Catalyst in the reaction of APG-12's synthesis played a key role. Catalyst which 4-toluene sulfonic acid and phosphoric acid compounded was used in this experiment. The molar ratio of 4-toluene sulfonic acid and phosphoric acid had impact on product productivity in Figure 2.

As can be seen from Figure 2, the productivity which added phosphoric acid was also improved than before. No matter adding phosphoric acid or not, productivity firstly decreased, and then increased with the ratio increased. It is mainly because that excess acid may cause oxidation of glucose, and then make reactant carbonized. The analysis showed that the most appropriate ratio of toluene sulfonic acid and phosphoric acid is 3:1.

Although the conversion rate rises when it has a large amount of catalyst, the mount of catalyst is not the more the better. But if the catalyst dosage is so large, glucose may be generated by highly acidic catalyst. Different dosages of binary complex catalyst effects on the yield are shown in Figure 3.

We can see from Figure 3 that with the increase of catalyst dosage, reaction yield increased rapidly. It declined when reaching a certain amount. When the added amount is low, the catalytic performance of the catalyst

Table 1. Different types of catalysts influence on product characteristics.

\begin{tabular}{cccc}
\hline Catalyst type & number & Clarification time (min) & Yield (\%) \\
\hline p-toluenesulfonic acid & 1 & 112 & 80.9 \\
Concentrated sulfuric acid & 2 & 151 & 78.6 \\
p-toluenesulfonicacid+Phosphorous acid & 3 & 105 & 94.8 \\
Concentrated sulfuric acid+Phosphorous acid & 4 & 122 & 92.4 \\
p-toluenesulfonicacid+concentrated sulfuric acid & 5 & 115 & 89.2 \\
\hline
\end{tabular}

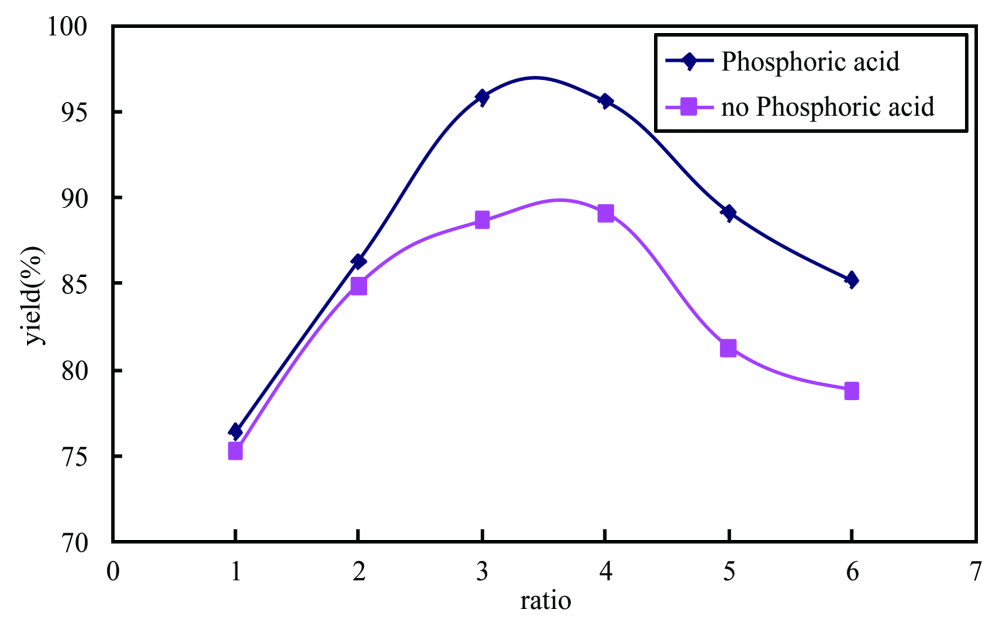

Figure 2. The effect of compound binary catalyst to the yield. 
is weak. The reaction rate is slow, which results in a low yield. So, the dosage of the catalyst must ensure that the joined glucose can be consumed rapidly. Otherwise, glucose will gradually accumulate. It aggregated because reaction is too late. Particle size became bigger. The reaction is more difficult. When the added amount is great, the reaction rate accelerates; then, glucose is prone to aggregation, too. The analysis drawn that complex catalyst at $1.3 \%$ is best. It has high yield and fast reaction rate at this time.

\subsection{The Effect of Reaction Temperature}

To discuss the influence of temperature to yield, we do experiment with set different temperature gradients under the conditions of the molar ratio of alcohol and glucose 7:1, binary catalyst dosage $1.3 \%$ and pressure $4 \mathrm{kPa}$. In the case of other conditions unchanged, we set nine reaction temperature points respectively at $90^{\circ} \mathrm{C}, 95^{\circ} \mathrm{C}$, $95^{\circ} \mathrm{C}, 105^{\circ} \mathrm{C}, 110^{\circ} \mathrm{C}, 115^{\circ} \mathrm{C}, 120^{\circ} \mathrm{C}, 125^{\circ} \mathrm{C}$ and $135^{\circ} \mathrm{C}$. We conclude the best reaction temperature. The experimental data are shown in Table 2.

When temperature is below $90^{\circ} \mathrm{C}$, the production rate is extremely low, only about $20 \%$. When the reaction temperature rises to $105^{\circ} \mathrm{C}$, the yield increases to over $90 \%$ rapidly. It suggests that the reaction takes place smoothly under the condition of temperature above $105^{\circ} \mathrm{C}$. When temperature at $90^{\circ} \mathrm{C}$ to $100^{\circ} \mathrm{C}$, the reaction liquid color almost doesn't change and it is still white suspension. When the temperature is $120^{\circ} \mathrm{C}$, the reaction takes place smoothly. The reaction time shortens for an hour, but the product color becomes orange-yellow. When the reaction temperature is high, the production cost increases and the product yield decreases. When the temperature is $125^{\circ} \mathrm{C}$, a lot of black material generates. By inference, it is caused by glucose carbonization at high temperature. Yield drops to $93 \%$ at $135^{\circ} \mathrm{C}$. Therefore, to obtain a higher quality of APG-12, the choice of temperature is extremely important. Generally, synthetic reaction temperature control in $110^{\circ} \mathrm{C}-120^{\circ} \mathrm{C}$ is appropriate.

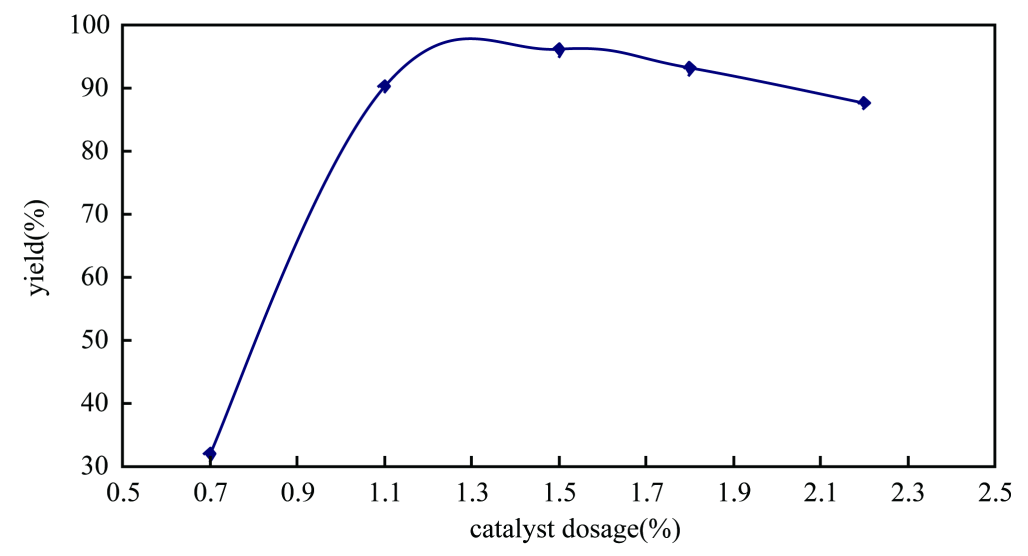

Figure 3. The influence of catalyst dosage on the yield of APG-12.

Table 2. The reaction temperature impact on the yield and color of were APG-12.

\begin{tabular}{cccc}
\hline Reaction temperature $\left({ }^{\circ} \mathrm{C}\right)$ & Color & Reaction time (h) & Yield (\%) \\
\hline 90 & White & 4.0 & 4.0 \\
95 & White & 4.0 & 30.5 \\
100 & White & 4.0 & 65.0 \\
105 & Faint yellow & 4.0 & 98.0 \\
110 & Faint yellow & 4.0 & 98.1 \\
115 & Faint yellow & 3.0 & 98.4 \\
120 & Orange-yellow & 2.5 & 98.0 \\
125 & Black & 2.5 & 95.3 \\
\hline
\end{tabular}




\subsection{The Effects of Reaction Pressure}

The change of pressure would affect dehydration rate. Due to the synthetic reaction, it is a reversible reaction. Reduced water volume of resultant is conducive to the positive direction of motion in reaction to improve the rate of reaction product. The higher temperature is, the lower pressure is and the faster dehydration speed is. Under the condition of molar ratio of sugar alcohol 7:1, the temperature was $115^{\circ} \mathrm{C}$. The amount of catalyst was 1.3\%. Effect of yield was observed by changing reaction pressure. The experimental result is shown in Figure 4.

As can be seen from the Figure 4, the lower the pressure is, the higher yield is. When the pressure is greater than $8 \mathrm{kPa}$, yield decreases to $20 \%$ or less. In the $4 \mathrm{kPa}-8 \mathrm{kPa}$, yield changes rapidly. Yield comes into balance when the pressure is less than $4 \mathrm{kPa}$. Productivity reached over $90 \%$. Therefore, the best reaction pressure should be $3 \mathrm{kPa}-4 \mathrm{kPa}$.

\subsection{Characterization of APG-12}

Characterization of the APG-12 was analyzed by infrared spectroscopy. Infrared spectroscopy is common and easy way to analyze and determine the structure of synthetic production. Figure 5 is APG-12 synthesized in the infrared absorption spectrum of the production.

Different substances have different bonds. The absorption wavelength is different. Functional groups of production could be inferred by the absorption peak attribution. As can be seen from Figure 5, in $1710.4 \mathrm{~cm}^{-1}$, this synthetic product has a feeble $\mathrm{C}-\mathrm{O}-\mathrm{C}$ structure stretching vibration peak which could judge whether has a type of APG-12 surfactant material generated or not. Analysis shows that the product is APG-12.

\section{Conclusion}

This experiment synthesized APG-12 by the direct glycosidation (one-step). The technological process is simple

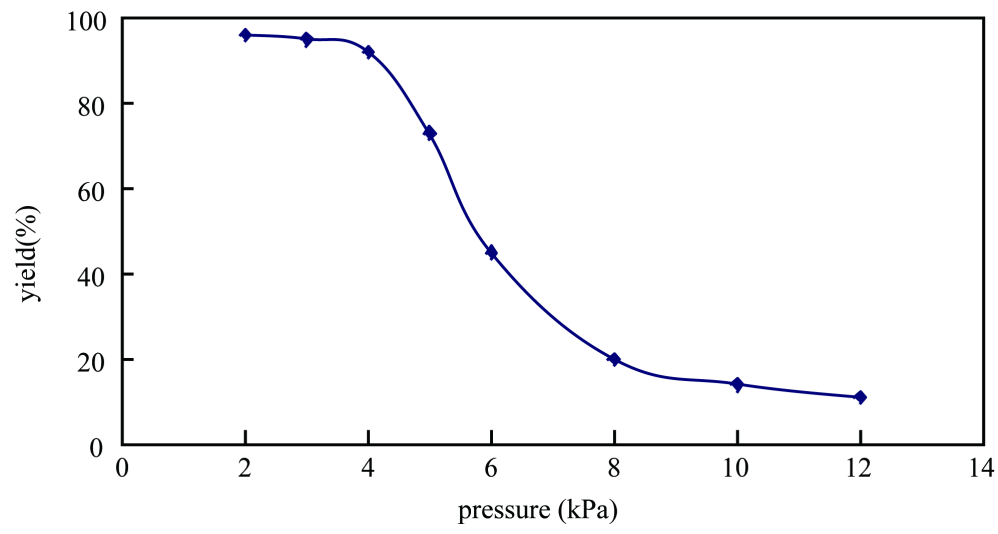

Figure 4. The influence of pressure to the yield.

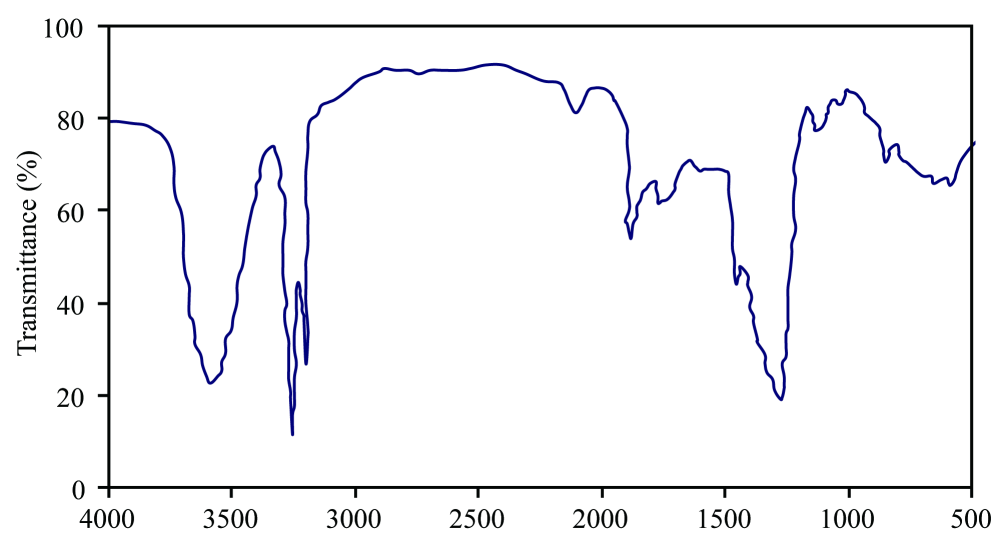

Figure 5. The infrared spectra of APG-12. 
and the yield is high. The binary catalyst systems of toluene-p-sulfonic acid and ortho-phosphoric acid were synthetized indoor and determined that the best mole ratio was 7:1 and that dosage was $1.3 \%$. Through the analysis of the optimization and impact conditions of APG-12 synthesis, the best reaction process conditions are: A mole ratio of lcohol and glucose is $7: 1$; system reaction temperature is $115^{\circ} \mathrm{C}$; binary catalyst dosage is $1.3 \%$ and pressure control is 3 - $4 \mathrm{kPa}$. Analyzing the product by using infrared spectroscopy, it has a feeble C-O-C structure stretching vibration peak. So, we can determine that the product is APG-12.

\section{Acknowledgements}

The project is supported by Heilongjiang Province Natural Science Foundation of Cationic Gemini Surfactant Study of Oil Displacement Technique. Fund No.: E201337.

\section{References}

[1] Wu, M.H., Chen, H.Q., et al. (2000) The Profile Technology of Synthetize Alkyl Polyglycoside. Chemical Engineering, 25, 52-54.

[2] Lu, W.J. (2005) The Synthesis and Application of Alkyl Poly Glucoside. Chemical Industry in Guangxi, 26, 32-39.

[3] Li, H.P., Wang, X.J., C.Y., et al. (2007) The Research on Synthesis And Performance of APG-12. Journal of Zhengzhou Grain College, 6, 76-85.

[4] Dai, F.G. and Rong, H.C. (1997) Domestic and Foreign Research and Industrial Production on Alkyl Polyglycoside (APG). The Surfactant Industry, 4, 5-7.

[5] Yu, N. (2004) Synthesis and Analysis of Physical and Chemical Properties of Alkyl Glucosides. Master's Thesis, Dalian University of Technology, Dalian, 20-34. 
Scientific Research Publishing (SCIRP) is one of the largest Open Access journal publishers. It is currently publishing more than 200 open access, online, peer-reviewed journals covering a wide range of academic disciplines. SCIRP serves the worldwide academic communities and contributes to the progress and application of science with its publication.

Other selected journals from SCIRP are listed as below. Submit your manuscript to us via either submit@scirp.org or Online Submission Portal.
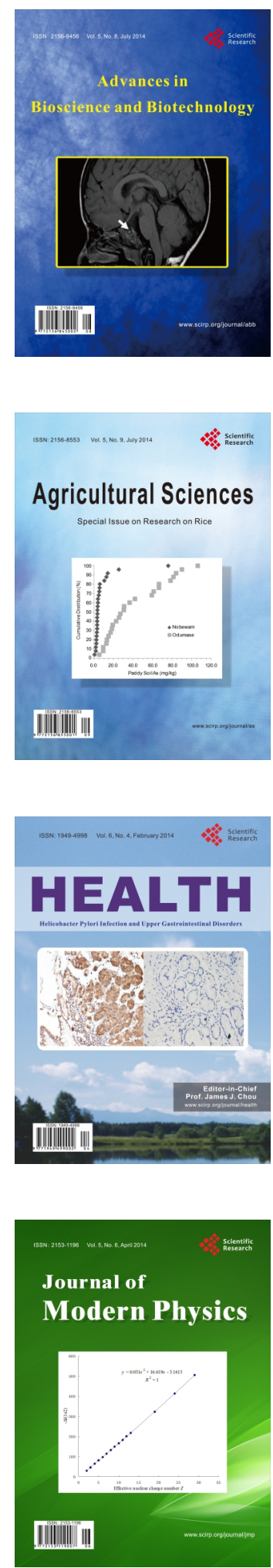
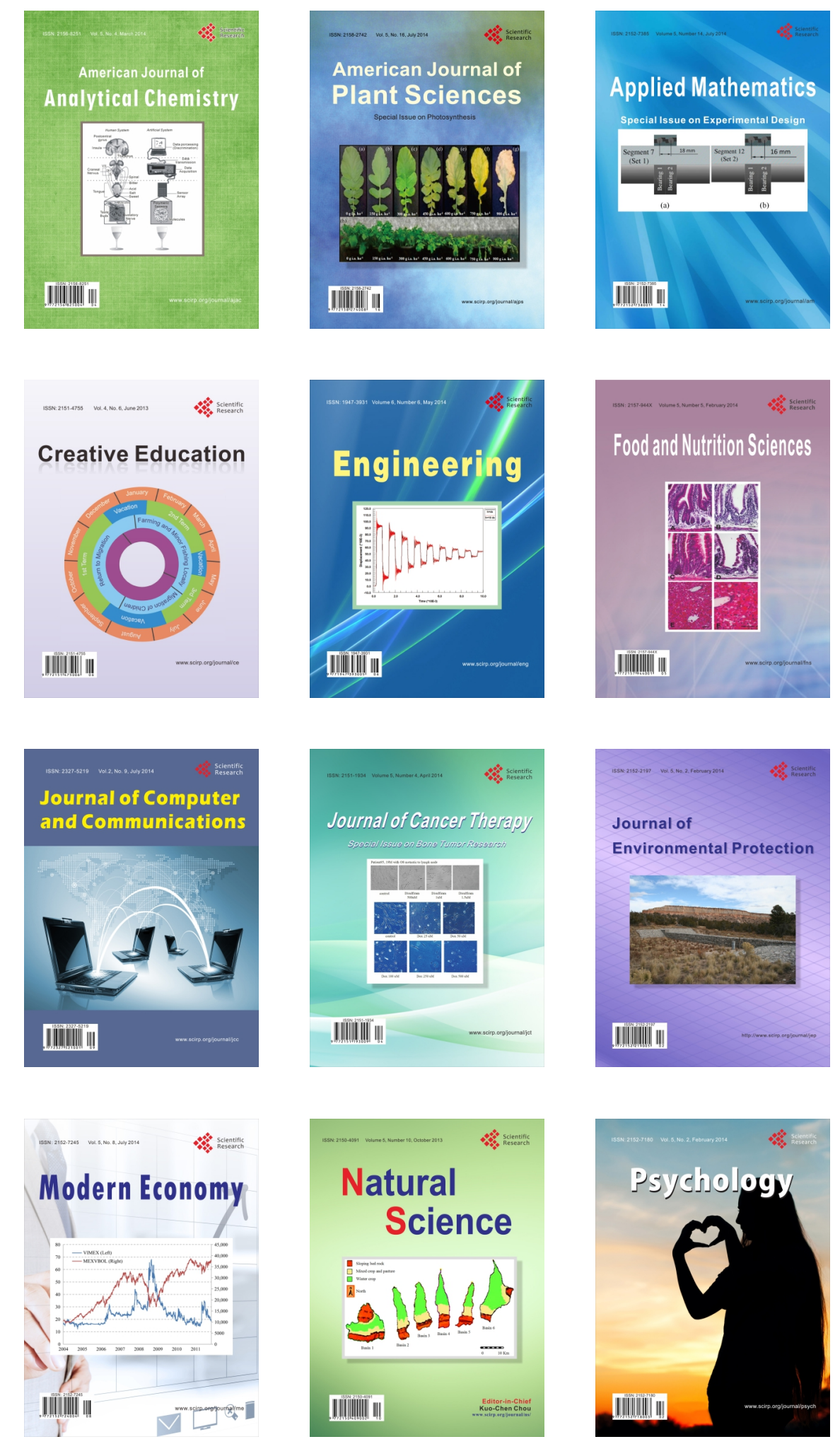